\title{
Diagnostic Value of Novel Biomarker Human Epididymis Protein 4 (HE4) in Detecting Endometrial Cancer
}

\author{
Mehri Jafari-Shobeiri ${ }^{1}$, Marzye Jangi ${ }^{1 *}$, Ali Dastranj Tabrizi ${ }^{1}$, Manizheh Sayyah-Melli ${ }^{1}$, Parvin Mostafa-Gharabaghi ${ }^{1}$, \\ Elaheh Ouladsahebmadarek ${ }^{1}$, Esmail Neginfar ${ }^{2}$, Yasmin Pouraliakbar $^{3}$
}

\begin{abstract}
Objectives: Endometrial cancer (EC) is the most common malignancy of the female reproductive system. To date, no good marker for screening or disease monitoring of $\mathrm{EC}$ is available. The objective of this study was to investigate the sensitivity and specificity of CA125 and human epididymis protein 4 (HE4) in detection of EC.

Materials and Methods: This case-control study was carried out on 40 women with EC and 60 women without cancer. Serum samples were prospectively obtained from all patients. Cut-off points for HE4 and CA125 were considered $70 \mathrm{pmol} / \mathrm{L}$ and $35 \mathrm{U} / \mathrm{mL}$, respectively. The level of statistical significance is set at $P<0.05$.

Results: The ROC-AUC, for HE4 was 0.82 and CA125 was 0.73 and for combination of HE4 and CA125 was 0.89. Compared to CA125, HE4 had higher sensitivity ( $57.7 \%$ vs. $40 \%$ ), equal specificity ( $93.3 \%$ vs. $95 \%$ ), equal positive predictive value (PPV) ( $85 \%$ vs. $84.2 \%)$ and higher negative predictive value (NPV) (76.71\% vs. $70.37 \%)$ and in combination of two tumor markers sensitivity, specificity, PPV and NPV were $62.5 \%, 93.3 \%, 86.2 \%$ and $78.9 \%$, respectively. It was clear that combination of two markers had higher sensitivity and higher NPV to detect EC, than each marker alone. HE4 and CA125 were significantly elevated in EC compared to controls $(P<0.001)$. There was significant correlation between median HE4 and age of the patients $(\mathrm{r}=0.48, P=0.002)$ and stage of the disease $(\mathrm{r}=0.50, P=0.001)$. There was no significant correlation between CA125 and age, stage or grade of the disease $(P=0.39$, $P=0.08$ and $P=0.9$, respectively).

Conclusion: Our study showed that serum HE4 levels alone and in combination with CA125 are sensitive markers in diagnosing EC. Keywords: CA125 protein, Endometrial neoplasms, HE4 protein, Laboratory diagnosis
\end{abstract}

\section{Introduction}

Endometrial cancer (EC) is one of the most common malignancies of the reproductive system in the world. It is the fourth most prevalent cancer after breast, lung and colorectal cancers and the eighth leading cause of death from cancer in women. The risk of EC during a woman's life is $2 \%-3 \%$ (1) and seems to be more with the development of obesity (2) and occurring at younger ages than previous (3).

Ninety percent of uterine cancers arise from endometrium and are classified as endometrial adenocarcinoma (EAC), $80 \%$ of which are low grade endometrioid cancer and are detected at lower stages of the disease. It has a good prognosis, is recognized as type I EC, and is usually diagnosed early due to bleeding after menopause. In contrast, type II, which includes the remaining $20 \%$, shows other histological signs such as clear and serous cells. It develops with invasion with fast metastasis in the early stages of the disease and has a poor prognosis (2). 13\%-17\% of patients experience recurrence after treatment which often happens in the first 3 years. The 3 -year survival following relapse is
$73 \%$ for vaginal recurrence and less than $15 \%$ for recurrence in pelvis and other parts. In addition, relapse occurs in $60 \%$ of low-risk patients (with endometrioid histology and low grade or stage). Half of these patients develop extensive relapse and have poor prognosis (3).

Although EC can be diagnosed at an early stage according to the common symptom of vaginal bleeding, its detection at more advanced stages is associated with poor prognosis (4).

Given the high incidence of the disease, decreased age of diagnosis, and unfavorable prognosis in high-risk patients, it seems necessary to identify the high-risk patients before surgery and to perform disease screening using a tumor marker which can help to identify high-risk cases, to choose better surgical techniques and to increase survival. On the one hand, it may help prevent invasive surgery and increased mortality in patients with limited disease and assist in post-treatment follow-up and identification of recurrence.

CA125 levels increase in many primary tumors such as ovarian, endometrial, colorectal, breast, and lung cancers. 
It also increases in other conditions such as pregnancy, inflammation, endometriosis, fibrosis, benign ovarian cysts, cirrhosis, and abdominal surgery (5).

Serum CA125 rises in $10 \%-20 \%$ of patients with early stages of EC and only in $25 \%$ of asymptomatic patients with recurrence. Measurement of CA125 is highly performed in the diagnosis of advanced stages EC (6-9).

Although human epididymis protein 4 (HE4) exists in normal tissues such as epithelium of mammary glands, female genital tract, vas deferens, respiratory glands, colonic mucosa, and salivary glands, its serum titers increase in cancer tissues, such as mesothelioma and lung, endometrial, breast, digestive tract, and interstitial tissue cancers; however, the highest serum HE4 levels are seen in ovarian cancer in women and lung cancer in men (10). In 2009, HE4 was accepted as biomarker for prognosis and follow-up of recurrent ovarian epithelial cancer by Food and Drug Administration (FDA) (2).

There is increasing evidence demonstrating that the novel factor HE4 can be a marker for EC. HE4 is increased in all stages of EC and is more sensitive than CA125 for detection of early stage EC. Further research confirms the role of HE4 as a marker of recurrence in early-stage, and during the follow-up of response to treatment (11). Another study showed that HE4 reduced after initial treatment of EC and increased again following recurrence (3). $\mathrm{HE} 4$ can be useful in identifying high-risk patients before surgery $(12,13)$.

The present study aimed at determining preoperative serum levels of CA125 and HE4 in patients with EC as well as determining sensitivity and specificity of these tumor markers in the diagnosis of EC.

\section{Materials and Methods}

This case-control, descriptive-analytical study was performed from March 2013 until December 2014 on surgical candidates admitted to the Surgery and Oncology ward of Al-Zahra hospital. According to the pilot study in which the sensitivity of HE4 in the diagnosis of EC was obtained $95 \%$, and assuming $\alpha=0.05$, power $=80 \%$, and $10 \%$ difference in diagnostic accuracy, a total of 40 patients with EC was estimated as the sample size and 60 subjects were also selected as the controls that were matched with EC patients in terms of age.

Patients were selected from women diagnosed with EC (based on curettage specimens or outpatient endometrial biopsy) who were hospitalized for surgical hysterectomy in Al-Zahra hospital, after obtaining medical observation, performing physical examination, filling out the questionnaire, and taking informed consent. Controls consisted of patients who were admitted for hysterectomy because of non-cancer reasons such as myoma or bleeding, after obtaining medical history, performing physical examination, filling out the questionnaire, and taking informed consent. All patients were informed of the purpose of the study and written consent was obtained.

Exclusion criteria were previous history of cancer in any part of the body, prior radiation or chemotherapy, metastatic tumors of the uterus, diabetes, hepatic cirrhosis or dysfunction (bilirubin above $1.5 \mathrm{mg} / \mathrm{dL}$ ), renal dysfunction (creatinine above $1.5 \mathrm{mg} / \mathrm{dL}$ ), and pregnancy.

The diagnosis of EC and benign lesions were confirmed histologically by senior gynecologic pathologist. All patients with EC were staged and graded according to the recommendations of International Federation of Gynecology and Obstetrics (FIGO).

Five milliliters venous blood sample was collected the day before surgery from each patient. Blood samples were immediately centrifuged at $3000 \mathrm{rpm}$ for 10 minutes to extract the serum at $4^{\circ} \mathrm{C}$. The serum was stored at $-80^{\circ} \mathrm{C}$ until analysis were performed. The time interval between sampling and freezing was up to 1 hour.

Serum HE4 was measured by electrochemiluminescence enzyme immunoassay (Roche, Germany) and serum CA125 by chemiluminescence enzyme immunoassay (Roche, Germany). Cut-off points for HE4 and CA125 were considered $70 \mathrm{pmol} / \mathrm{L}$ and $35 \mathrm{U} / \mathrm{mL}$, respectively.

\section{Statistical Analysis}

Frequency and percentage were used to describe the qualitative data and mean \pm standard deviation or median and interquartile range quantitative data. Given the skewness and asymmetry of the data, median and interquartile range were used to describe data when reporting the results of serum tumor markers in both groups.

The relationship between the biomarkers and age was analyzed through non-parametric Spearman correlation coefficient and the relationship between the biomarker and disease grade and stage through Kruskal-Wallis test. Mann-Whitney test was used to compare biomarkers between the control group and the patients.

To determine the diagnostic accuracy of biomarkers for EC, sensitivity, specificity, positive predictive value (PPV), negative predictive value (NPV) and the area under the receiver operator characteristic (ROC) curve was calculated. The statistical analysis was carry out by SPSS 17 software and $P$ values less than 0.05 were considered statistically significant.

\section{Results}

Serum samples were obtained from 40 patients with surgically staged EC and from 60 patients with benign lesions. The clinical characteristics of both groups were presented in Table 1. Serum levels of HE4 and CA125 were increased significantly in the patient group, compared with the control group (Table 2).

To evaluate the utility of preoperative tumor markers in predicting EC, sensitivity and specificity calculations were performed. The cut-offs were $70 \mathrm{pmol} / \mathrm{L} \mathrm{HE} 4$ and $35 \mathrm{U} /$ $\mathrm{mL}$ for CA125. The sensitivity, specificity, PPV, NPV and area under the ROC curve of the HE4 were $57.7 \%, 93.3 \%$, $85.1 \%, 76.71 \%$ and $0.82 \%$ (CI: $0.73-0.91$ ), respectively (Figure 1). The sensitivity, specificity, PPV, NPV and area under the ROC curve of the CA 125 were $40 \%, 95 \%, 84.2 \%$, 
Table 1. The Clinical Characteristics of the Patient and the Control Groups

\begin{tabular}{|c|c|c|c|}
\hline & $\begin{array}{l}\text { Endometrial Cancer Group } \\
\qquad(\mathrm{n}=40)\end{array}$ & $\begin{array}{c}\text { Control Group } \\
(n=60)\end{array}$ & $P$ Value \\
\hline Median age (range) & $56(32-76)$ & $50.5(32-76)$ & 0.14 \\
\hline Menopause (\%) & $31(77.5)$ & $30(50)$ & 0.0006 \\
\hline Oral contraceptive pill use (\%) & $5(12.5)$ & $11(18)$ & 0.47 \\
\hline Infertility (\%) & $7(17.5)$ & $2(3.3)$ & 0.03 \\
\hline \multicolumn{4}{|l|}{ FIGO stage (\%) } \\
\hline I & $21(52.5)$ & - & \\
\hline ॥ & $13(32.5)$ & - & \\
\hline III & $6(15)$ & - & \\
\hline IV & 0 & - & \\
\hline \multicolumn{4}{|l|}{ Tumor grade (\%) } \\
\hline 1 & $12(30)$ & - & \\
\hline 2 & $13(32.5)$ & - & \\
\hline 3 & $15(37.5)$ & - & \\
\hline \multicolumn{4}{|l|}{ Histology (\%) } \\
\hline Endometrioid adenocarcinoma & $35(87.5)$ & - & \\
\hline Non-endometrioid carcinoma & $5(12.5)$ & - & \\
\hline
\end{tabular}

Table 2. Serum Levels of HE4 and CA125 in the Patient and the Control Groups

\begin{tabular}{lccc}
\hline & $\begin{array}{c}\text { Endometrial } \\
\text { Cancer Group } \\
(\mathbf{n = 4 0 )}\end{array}$ & $\begin{array}{c}\text { Control Group } \\
(\mathbf{n}=60)\end{array}$ & P Value \\
\hline HE4 (pmol/l) & 81.68 & 40.32 & \\
$\quad$ Median & $50.49-144.55$ & $32.77-50.32$ & $<0.001$ \\
Quarter 1- Quarter 3 & & & \\
CA125 (U/L) & 24.79 & 10.29 & \\
Median & $10.84-85.2$ & $7.34-18.73$ & $<0.001$ \\
Q1-Q3 & & & \\
\hline
\end{tabular}

70.37\% and $0.73 \%$ (CI: $0.63-0.83$ ) respectively (Figure 1 ). In combination of two tumor markers sensitivity $62.5 \%$, specificity $93.3 \%$, PPV $86.2 \%$, NPV $78.9 \%$ and ROC-AUC was 0.89 (CI: 0.82-0.95).

A positive correlation existed between age and HE4 levels in the patient group (patients, $\mathrm{r}=0.48, P=0.002$ ). There was also a positive but statistically insignificant correlation between serum HE4 and grade of the disease in the patient group $(\mathrm{r}=0.28, P=0.07)$.

In addition, a significant positive correlation existed between serum HE4 and stage of the disease in patients $(P=0.001, r=0.50)$. There was a significant negative correlation between age and level of CA125 in the control group $(P=0.007, \mathrm{r}=0.34)$. No significant association existed between serum levels of CA125 and age, and disease stage and grade $(P=0.08, P=0.9, P=0.39$, respectively) (Table 3).

\section{Discussion}

EC is the most common gynecological malignancy in the Western world. Unfortunately, to date, no good marker for EC screening, early diagnosis or disease monitoring is available (14). A sensitive marker is needed that can detect EC in its early or advance stages or detect its recurrence. In this study, serum levels of HE4 and CA125 were high in patients with EC; therefore, HE4 can help as a new marker in diagnosing this disease. In our study HE4 is more sensitive than CA125 in distinguishing EC patients from women with benign lesions in uterus, regardless of tumour stage and grade. Zhang and Zhang noticed that serum levels of tumor markers in patients with EC were higher compared to healthy women and patients with benign diseases (15).

In our study all patients with EC, were surgically staged. Most of the patients were in stage one of the disease. HE4 was a better marker for detecting both the early and advanced stages of EC compared to CA125. In the research with median serum HE4 levels of $83 \mathrm{pm}$ and $79.9 \mathrm{pm}$, there was a correlation between disease stage and HE4 serum level $(14,16)$. In this study, this correlation existed at median serum HE4 level of $81.68 \mathrm{pm}$.

Some researchers found that disease grades and HE4 serum levels were correlated $(14,17)$, which disagrees with the findings of our research. However, Mutz-Dehbalaie et al (18) conducted research on 183 patients and found no correlation between tumor grades and serum HE4 levels.

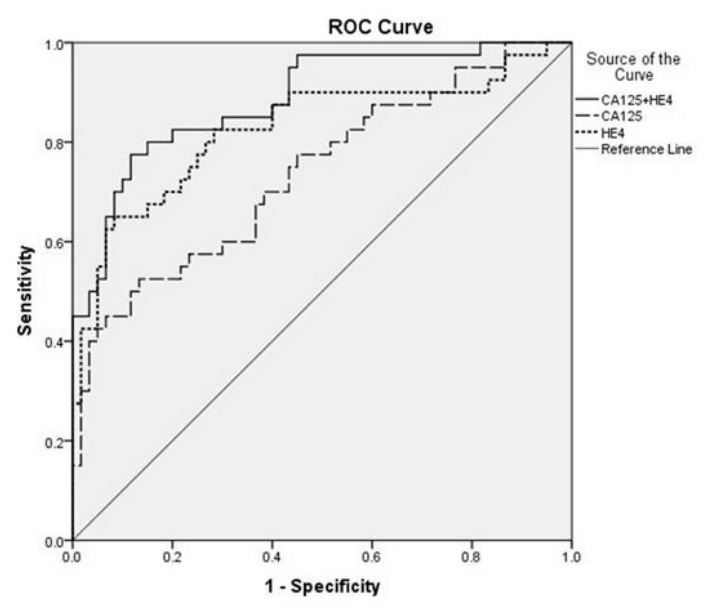

Figure 1. ROC Curve to Confirm the Diagnostic Value of HE4 and CA125 and CA125+HE4 in Endometrial Cancer. 
Table 3. Serum Levels of HE4 and CA125 in the Patients With Endometrial Carcinoma According to the Age, Stage and Tumor Grade

\begin{tabular}{|c|c|c|c|c|}
\hline & HE4 (PMOL/L) Median (Q1-Q3) & $P$ Value & CA125 (U/L) Median (Q1-Q3) & $P$ Value \\
\hline \multicolumn{5}{|l|}{ Age } \\
\hline$\leq 56$ & $61.32(48.13-112.56)$ & & $23.61(12.25-111.02)$ & \\
\hline$>56$ & 129.45 (71.94-258.9) & 0.014 & $24.79(10.2-80.4)$ & 0.73 \\
\hline \multicolumn{5}{|l|}{ Grade } \\
\hline 1 & $78.87(43.72-144.55)$ & & $30.30(11.19-96.92)$ & \\
\hline 2 & $54.19(49.95-102.88)$ & & $20.87(11.45-64.34)$ & \\
\hline 3 & $113.5(75-282)$ & 0.077 & $25.18(7.19-137)$ & 0.84 \\
\hline \multicolumn{5}{|l|}{ Stage } \\
\hline I & $68.28(43.74-106.8)$ & & $12.77(10.48-40.95)$ & \\
\hline II & 125.9 (50.94-192.5) & & $55(21.38-170.15)$ & \\
\hline III & $401.5(106.37-616.02)$ & & $59.24(7.17-305.52)$ & \\
\hline IV & - & 0.005 & - & 0.054 \\
\hline
\end{tabular}

Angioli et al (17) studied 101 patients with ECs. Their average age was 64.9 years and their mean serum HE4 and CA125 levels were $128.07 \pm 120 \mathrm{pm}$ and $57 \pm 39.26 \mathrm{U} / \mathrm{L}$, respectively, which were lower than the levels we found in our study. Contrary to HE4, the serum levels of CA125 were higher in the patient group and there was a significant correlation between serum CA125 levels and the stage of the disease and the condition of lymph nodes. The patients were old and in advanced stages of the disease, and a large number of EC exhibited non-endometrioid histology, which was probably why these researchers found results different from ours.

Moor et al compared serum HE4, CA125, and CA72-4 levels in patients with endometrioid adenocarcinoma of the uterus and healthy menopausal women (the control). They noticed that HE4 was the most accurate marker for detecting EC regardless of tumor stage and that the area under the ROC curve for HE4 (ROC-AUC) in the first, second to fourth, and all stages were higher (76.7, 83.6 and 78.7, respectively) compared to the other markers (11).

In our study, HE4 had higher sensitivity than CA125 alone. Adding CA125 to HE4 made them more sensitive compared to HE4 and CA125 alone. When these two markers were used together, they had an ROC-AUC of 0.89 , which was higher than that of HE4.

In our study, HE4 had higher sensitivity than CA125 in detecting EC. ROC-AUC of HE4 was 0.82, which was higher than the 0.73 for CA125. The combined detection of serum HE4 and CA125 increased the ROC-AUC to 0.89 , which was higher than the figure for each of them when used alone.

The serum level of CA125 in patients with EC was significantly higher compared to the control group and contrary to HE4, there was no correlation between serum CA125 levels and the age of the patients or the stage or grade of the disease. In a study conducted on 193 patients with EC with median CA125 serum level of $117.2 \mathrm{U} / \mathrm{L}$, there was a significant correlation between the CA125 serum level and the degree of lymph node invasion (16). The reasons for the differences between the two studies could probably be the small sample sizes and the low average age of the patients in our study.

\section{Conclusion}

EC is a common disease that, despite the possibility of its early detection, is diagnosed at advanced stages in some patients. Moreover, high risk patients need extensive surgery and this invasive treatment can reduce survival rate in patients in whom the disease has not spread widely. Therefore, it is important to identify patients who benefit from these treatments. HE4 is a new tumor marker that can prove useful in earlier detection of the disease, identification of high-risk patients, follow-up after treatment, and anticipating survival rates.

In this study, serum levels of the two tumor markers (HE4 and CA125) were higher in some EC patients; and contrary to CA125, there was a significant correlation between the age of the patient and the stage of the disease when HE4 was used. Study of the diagnostic values of these two tumor markers revealed that HE4 had higher sensitivity for EC compared to CA125, and that the combination of these two tumor markers could detect this disease better than HE4 alone.

Further investigation is warranted to assess the potential of HE4 as a new tool for prospective diagnosis of EC patients

\section{Ethical issues}

Ethical approval was obtained from the Research Vice Chancellor Office, Tabriz University of Medical Sciences.

\section{Conflict of interests}

The authors have no conflicts of interest to disclose.

\section{Financial support}

This study was supported by Women's Reproductive Health Research Center, Tabriz University of Medical Sciences.

\section{Acknowledgments}

We would like to thank authorities of Tabriz University of Medical Sciences for the scientific and ethical approval and financial support of this research. This study has been done as a thesis for specialty degree of Marzye Jangi in Women's Reproductive Health Research Center, Tabriz 
University of Medical Sciences.

\section{Reference}

1. Dowdy S, Mariani A, Lurain J. Uterine Cancer. In: Berek JS, Berek DL, Hengst GB, eds. Berek\& Novak's Gynecology. 15th ed. Philadelphia: Lippincott Williams \& Wilkins; 2012:1250-2.

2. Brennan DJ, Hackethal A, Metcalf AM, et al. Serum HE4 as a prognostic marker in endometrial cancer--a population based study. Gynecol Oncol. 2014;132(1):159-165. doi:10.1016/j. ygyno.2013.10.036.

3. Brennan DJ, Hackethal A, Mann KP, et al. Serum HE4 detects recurrent endometrial cancer in patients undergoing routine clinical surveillance. BMC Cancer. 2015;15:33. doi:10.1186/s12885-015-1028-0.

4. Li J, Dowdy S, Tipton T, et al. HE4 as a biomarker for ovarian and endometrial cancer management. Expert Rev Mol Diagn. 2009;9(6):555-566. doi:10.1586/ erm.09.39.

5. Bie Y, Zhang Z. Diagnostic value of serum HE4 in endometrial cancer: a meta-analysis. World J Surg Oncol. 2014;12(1):169. doi:10.1186/1477-7819-12169.

6. Escudero JM, Auge JM, Filella X, Torne A, Pahisa J, Molina R. Comparison of serum human epididymis protein 4 with cancer antigen 125 as a tumor marker in patients with malignant and nonmalignant diseases. Clin Chem. 2011;57(11):1534-44. doi:10.1373/ clinchem.2010.157073.

7. Niloff JM, Klug TL, Schaetzl E, Zurawski VR Jr, Knapp RC, Bast RC Jr. Elevation of serum CA125 in carcinomas of the fallopian tube, endometrium, and endocervix. Am J Obstet Gynecol. 1984;148(8):10571058. doi:10.1016/S0002-9378(84)90444-7.

8. Brand JM, Frohn C, Cziupka K, Brockmann C, Kirchner H, Luhm J. Prolactin triggers proinflammatory immune responses in peripheral immune cells. Eur Cytokine Netw. 2004;15(2):99-104.

9. Yurkovetsky Z, Ta'asan S, Skates S, et al. Development of multimarker panel for early detection of endometrial cancer. High diagnostic power of prolactin. Gynecol Oncol. 2007;107(1):58-65. doi:10.1016/j.ygyno.2007.05.041.

10. Simmons AR, Baggerly K, Bast RC Jr. The emerging role of HE4 in the evaluation of epithelial ovarian and endometrial carcinomas. Oncology (Williston Park) 2013;27:548-556.

11. Moore RG, Brown AK, Miller MC, et al. Utility of a novel serum tumor biomarker HE4 in patients with endometrioid adenocarcinoma of the uterus. Gynecol Oncol. 2008;110(2):196-201. doi:10.1016/j. ygyno.2008.04.002.

12. Moore RG, Miller CM, Brown AK, Robison K, Steinhoff M, Lambert-Messerlian G. Utility of tumor marker HE4 to predict depth of myometrial invasion in endometrioid adenocarcinoma of the uterus. Int J Gynecol Cancer. 2011;21(7):1185-1190. doi:10.1097/ IGC.0b013e3182229ad8.

13. Kalogera E, Scholler N, Powless C, et al. Correlation of serum HE4 with tumor size and myometrial invasion in endometrial cancer. Gynecol Oncol. 2012; 124(2):270-5. doi:10.1016/j.ygyno.2011.10.025.

14. Bignotti E, Ragnoli M, Zanotti L, et al. Diagnostic and prognostic impact of serum HE4 detection in endometrial carcinoma patients. $\mathrm{Br} \mathrm{J}$ Cancer. 2011;104:1418-1425. doi:10.1038/bjc.2011.109.

15. Zhang AM, Zhang P. Clinical value of combined detection of serum human epididymal secretory protein $\mathrm{E} 4$ and $\mathrm{CA}(125)$ in the diagnosis of endometrial carcinoma. Zhonghua $\mathrm{Fu}$ Chan $\mathrm{Ke} \mathrm{Za}$ Zhi. 2012;47(2):125-128. [Chinese].

16. Zanotti L, Bignotti E, Calza S, et al. Human epididymis protein 4 as a serum marker for diagnosis of endometrial carcinoma and prediction of clinical outcome. Clin Chem Lab Med. 2012;50(12):21892198. doi:10.1515/cclm-2011-0757.

17. Angioli R, Plotti F, Capriglione S, et al. The role of novel biomarker HE4 in endometrial cancer: a case control prospective study. Tumour Biol. 2013;34(1):571-6. doi:10.1007/s13277-012-0583-0.

18. Mutz-Dehbalaie I, Egle D, Fessler S, Hubalek M, Fiegl H, Marth C, et al. HE4 is an independent prognostic marker in endometrial cancer patients. Gynecol Oncol. 2012;126(2):186-91. doi:10.1016/j. ygyno.2012.04.022.

Copyright $\odot 2016$ The Author(s); This is an open-access article distributed under the terms of the Creative Commons Attribution License (http://creativecommons.org/licenses/by/4.0), which permits unrestricted use, distribution, and reproduction in any medium, provided the original work is properly cited. 